The mild-mannered Asiatic Black bear (Selenarctos thibetanus) is commonly seen in coniferous and broad-leaved mixed forests, tropical rainforests and in the Himalayas of China. Many other animals and plants are covered in The Natural History of China by Z. Ji, Z. Guangmei, W. Huadong and $X$. Jialin. The volume illustrates the variety and beauty of China's natural history through the lavish use of colour photographs accompanying the text. Published by Collins, price $£ 14.95$.

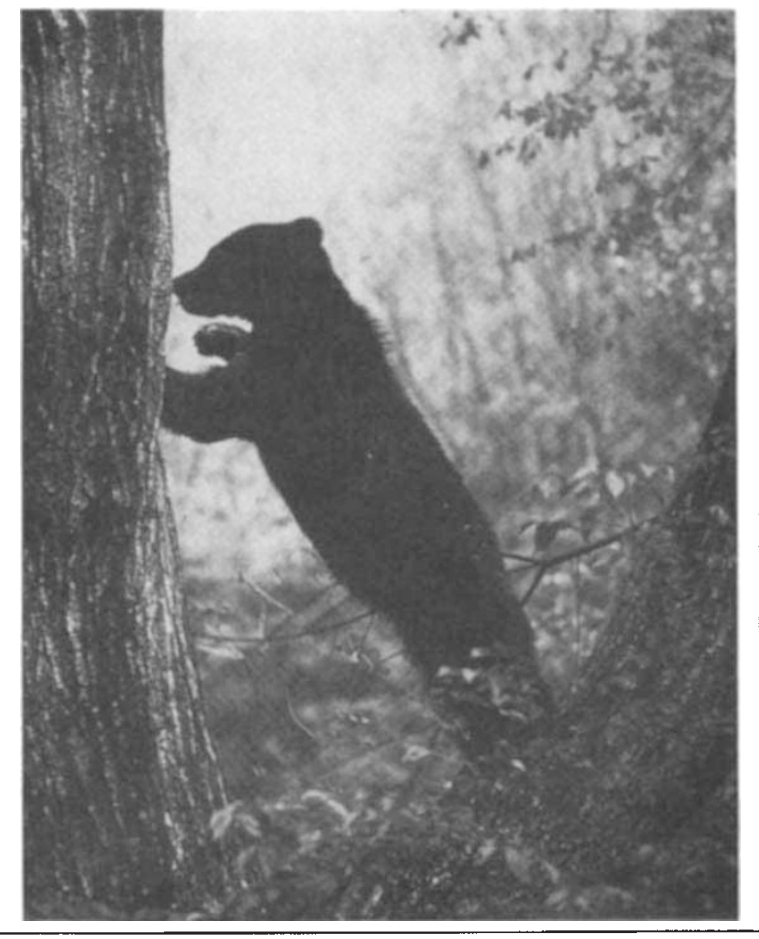

\section{Phylogeny of a pincushion}

\section{James W. Valentine}

Invertebrate Relationships: Patterns in Animal Evolution. By Pat Willmer. Cambridge University Press: 1990. Pp. 400. Hbk £32, \$49.50; pbk £12.95, \$22.95.

THE millions of living species of animals can be organized within a taxonomic hierarchy into about 35 phyla, each with distinctive body-plan features. At low hierarchical levels the relations among lineages are often fairly clear, but at increasingly higher levels, the taxa are separated by increasingly large morphological gaps, and their interrelations become correspondingly more speculative. The fossil record is of some help in closing those gaps, but the same principle applies, the relations among higher levels generally being the more enigmatic. Opinions among even the most masterful comparative morphologists have varied bewilderingly; the available evidence has not been a convincing guide to phylogenetic relationships at the scale of phyla, and quite a variety of competing hypotheses have been entertained.

Although phylogenies of the animal kingdom are given a chapter in textbooks, no book-length examination of the problems has appeared since Clark's admirable Dynamics in Metazoan Evolution in 1964. Now Willmer, in Invertebrate Relationships, provides a much-needed treatment that builds on that foundation, reviewing subsequent work and surveying the entire range of living phyla. It is a splendid book. In the early chapters, Willmer presents an overview of the previous hypotheses and recounts the chief sources of evidence for the phylogenetic schemes - comparative gross morphology, the fossil record, chemistry and genetics, embryology and ultrastructural studies. She then provides a review, phylum by phylum, of the principal ideas concerning their origins and relationships viewed in the light of that evidence. The accounts of supporting or contravening data are clear, documented together a widely scattered literature from many fields. In such a lengthy treatment the reader has the opportunity to return again and again from individual cases to the grand scheme of metazoan radiation, and thus to evaluate thoroughly the main schools of thought about the historical sequences and significance of such bodyplan features as the haemocoel, coelomic architectures and segmentation. Willmer's general conclusion is that these features are polyphyletic, and that the living bilaterian phyla, sometimes in groups of two to five but usually singly, can be traced back into a plexus of flatworm-like acoelomates. Non-bilaterians are traced back individually into planula-grade ancestors. The flatworm grade has arisen polyphyletically from the planulas, and the planulas polyphyletically from protozoans. Phylogenetic schemes that depict bilaterian phylogeny as a branching pattern based on primitive trimeric body plans (archicoelomates), or that depict the by a well-chosen bibliography that brings protostomes as primitive coelenterates or as primitive bilaterians, are rejected. The diagram illustrating the phylogenetic conclusions contains an empty box labelled "acoelomate platyhelminthes" from which 19 independent lineages proceed (3 of them questioned), looking like nothing so much as a pincushion. As to how the branching proceeded within the box to produce these 19 stocks - there are a few clues in the last chapter but no strong hypotheses. Such a pattern of independent origination of bilaterian lineages requires much convergent evolution, for which a principal support is found in the work of Manton (summarized in The Arthropoda, 1977).

It happens that I have been, for several years, writing a similar book that emphasizes evidence from the early fossil record, so perhaps it is unfair of me to say that I found the treatment of the fossils to be the least satisfactory part of this work. Willmer has missed the chance to support many of her contentions much more strongly, particularly her basic viewpoint that convergence abounds and that many architectural components are polyphyletic. On the other hand, she has not destroyed my own venture - there is still something left to be said. Indeed, now we have an up-to-date summing-up that presents, with greater clarity than any other of which I am aware, the implications and potential flaws in many of the competing phylogentic schemes. In Willmer's defence, the fossil literature is fairly new and (as in every other literature) scattered.

Also fairly new are the exciting molecular trees of animal phyla that have appeared too late for inclusion in this book. These trees are not inconsistent with an early branching of the ancestors of many living phyla among flatworm-grade organisms, and thus they may help to fill in the flatworm 'box' with details of the branching patterns. They also suggest, as Willmer gracefully concedes, that some of her placements of phyla will inevitably require changes (probably first among the annelids and lophophorates). The molecular work is still in a formative stage, however, and it is perilous to say much more at this stage.

Anyone with an interest in phylogeny at high taxonomic levels should own this book, and this applies to undergraduates in biological or palaeontological sciences as well as to advanced students and veteran professionals. It is an excellent sorting-out of a traditionally difficult area. We may be on the verge of a new level of understanding about the relationships among phyla, and this book is all the more valuable for that.

James $W$. Valentine is in the Department of Geological Sciences, University of California, Santa Barbara, California 93106, USA. 